\title{
Freiraum im Jugendalter - ein theoretischer und empirischer Versuch
}

\author{
Katrin Peyerl • Ivo Züchner
}

Eingegangen: 29. September 2019 / Angenommen: 20. April 2020 / Online publiziert: 26. Oktober 2020 (C) Der/die Autor(en) 2020

Zusammenfassung Der Beitrag unternimmt eine inhaltliche Bestimmung des schillernden Begriffs „Freiraum“, der im Kontext des Heranwachsens junger Menschen wieder Konjunktur hat. Hierzu wird ein theoretisches Modell vorgeschlagen, das Freiraum über die Dimensionen Raum, Zeit und Selbstbestimmung konzipiert. Dieses Modell wird auch empirisch, anhand einer quantitativen Studie zum Aufwachsen Jugendlicher in Internaten, auf seine Tragfähigkeit geprüft. Der sehr breit den Alltag umfassende Charakter von Internaten wird dabei genutzt, um neben einer Relationierung dieser Dimensionen auch institutionelle, interaktionelle und personale Einflussfaktoren auf das Freiraumerleben zu prüfen, die sich hier stark auf das Internat verdichten.

Schlüsselwörter Freiraum $\cdot$ Jugend $\cdot$ Jugendphase $\cdot$ Internate $\cdot$ Selbstbestimmung

Dr. K. Peyerl $(\bowtie) \cdot$ Prof. Dr. I. Züchner

FB 21 - AG Außerschulische Jugendbildung, Philipps-Universität Marburg,

Wilhelm-Röpke-Straße 6b, 35039 Marburg, Deutschland

E-Mail: katrin.peyerl@staff.uni-marburg.de

Prof. Dr. I. Züchner

E-Mail: zuechner@staff.uni-marburg.de 


\title{
"Free space" in adolescence-a theoretical and empirical approach
}

\begin{abstract}
The paper examines the German term "Freiraum" (translated into "free space"), which is a point of reference in the concerns of youth growing up in between institutions. We propose a theoretical model, which defines "Freiraum" dependent upon time, space and self determination. This model is tested with data from the study "growing up in boarding schools", where students were asked on their perception of free space in boarding schools. The results suggest a first validation of this model.
\end{abstract}

Keywords Free space $\cdot$ Boarding schools $\cdot$ Youth $\cdot$ Self determination

Der Begriff Freiraum hat beim Thema Jugend derzeit Konjunktur. Dabei waren Freiräume und sind immer schon eine Figur in der Beschreibung der Jugendphase, u. a. in der Entstehung von Jugend als eigenständiger Lebensphase, beim Wandervogel sowie in späteren jugendtypischen Aktivitäten und Gesellungsformen (Ferchoff 2006; Giesecke 1981). Ebenso findet er sich in theoretischen Beschreibungen des Heranwachsens (Zinnecker 2000) und zuletzt vermehrt in jugendpolitischen und jugendpädagogischen Forderungen, die mit einer Diagnose fehlender Freiräume für Jugendliche einhergehen (u.a. Deutscher Bundestag 2017; Arbeitsgemeinschaft für Kinder- und Jugendhilfe (AGJ) 2016). Mit diesem Beitrag soll der Versuch unternommen werden, Freiraum näher zu bestimmen und gleichzeitig Faktoren zu identifizieren, welche das Erleben von Freiraum im Jugendalter beeinflussen.

Der theoretische Zugang wird dabei durch einen empirischen Zugang ergänzt, indem die Perspektiven von Jugendlichen auf Freiraum auf der Grundlage der Daten einer quantitativen Studie zum Aufwachsen von Jugendlichen in Internaten (Züchner und Peyerl 2018) analysiert werden. Nun gelten Internate nicht unbedingt als Orte von Freiraum, aber gerade die institutionelle Geschlossenheit, ihre oft eher abgeschiedene Atmosphäre (Gibson 2017, S. 16) und die fließenden Grenzen zwischen schulischer und außerschulischer Lebenswelt (Ladenthin 2009) reduzieren die Vielfalt möglicher Freizeitaktivitäten und sozialer Einbindungen und bieten damit eine besondere Chance, Freiraumerleben ,,unter Kontrolle“ vergleichbarer Alltagsgestaltungen der Bewohner*innen zu analysieren. Zudem wird das Leben der Bewohner*innen, das in den meisten Fällen auf einen Raum verdichtet ist, pädagogisch und konzeptionell umfassend strukturiert. Charakteristisch sind dabei neben Regeln insbesondere feste Tagesverläufe, strukturierte Lern- sowie gemeinsame Essens- und Schlafenszeiten (Haep 2015, S. 129). Die im 15. Kinder- und Jugendbericht als ,fortschreitende Institutionalisierung und pädagogische Inszenierung des Alltags junger Menschen“ (Deutscher Bundestag 2017, S. 22) beschriebene Entwicklung ist Internaten als charakteristisches Strukturmerkmal entsprechend schon immer inhärent. 


\section{Freiraum - theoretische Annäherungen}

\subsection{Zur Bedeutung von Freiräumen}

Die Bedeutung von Freiräumen wird, wie bereits eingangs skizziert, insbesondere im Kontext der Jugendphase immer wieder aus verschiedenen Perspektiven diskutiert. Auf der einen Seite finden sich eher politisch-deklarative Forderungen und Empfehlungen. So fordert die AGJ (2016) Akteur*innen der Kinder- und Jugendhilfe auf, ,sich mehr für das Öffnen von Freiräumen zur Persönlichkeitsbildung zu engagieren und anwaltschaftlich für junge Menschen im Prozess der Verselbstständigung einzutreten“ (AGJ 2016, S. 3), selbst weitere Freiräume zu schaffen und bei der Ermöglichung von Freiräumen in formalen Bildungskontexten zu unterstützen (AGJ 2016, S. 12 f.). Auch der Deutsche Bundesjugendring (DBJR) beschreibt Freiräume als pädagogisch-normatives Ziel, in dem er betont, dass ,junge Menschen [...] neben der notwendigen Anregung und Erziehung Freiräume als Räume ohne staatliche oder gesellschaftliche Vordefinition, in denen das Aufwachsen so wenig wie möglich von außen gesteuert oder normiert wird, [benötigen]“ (DBJR 2010, S. 2).

Aus jugendtheoretischer Perspektive lässt sich eine Auseinandersetzung mit Freiräumen in der Idee des Moratoriums finden (u. a. Zinnecker 2000; Erikson 1980). Jürgen Zinnecker (2000) definiert das pädagogische Moratorium als „eine spezifische lebensgeschichtliche ,Auszeit' für die Jüngeren [...], sichtbar gemacht in ausgewiesenen Zeiten, Räumen, Statuspositionen und Diskursen, die einen Rückzug auf Zeit aus bestimmten Verpflichtungen und Teilhaben der bürgerlichen Gesellschaft beinhaltet" (Zinnecker 2000, S. 37). Heinz Reinders (2003), der das Moratorium von der Transition unterscheidet, geht dabei davon aus, dass mit dem Moratorium eine Gegenwartsorientierung einhergeht, bei der insbesondere die Gleichaltrigen für Jugendliche von Bedeutung sind. Verbunden damit ist für ihn weiterhin, dass Jugendliche sich Räume schaffen, in denen sie ,der Eigenständigkeit der aktuellen Lebensphase Ausdruck verleihen können" (Reinders 2003, S. 114) und sich somit eigene Sozialräume erschließen (Reinders 2003, S. 114f.), worin sich letztlich die Idee von Freiräumen abbildet. Die Sachverständigenkommission des 15. Kinderund Jugendberichts hat demgegenüber 2017 das Moratoriumskonzept als nur noch begrenzt tragfähig bewertet und beschreibt die Jugendphase aufgrund starker gesellschaftlicher Einbindung sowie vielfältiger gesellschaftlicher Erwartungen an junge Menschen eher als Vergesellschaftungsphase denn als Freiraum (Deutscher Bundestag 2017, S. 89 ff.). Ähnliches betont Lothar Böhnisch (2012), der von einem sich zunehmend auflösendem Moratorium ausgeht, ,je stärker soziale Bewältigungsprobleme in das Schulalter hineinreichen und sich die Unbekümmertheit jugendlichen Experimentierens mit sozialen Bewältigungsproblemen zu frühen biografischen Risiken vermischt“" (Böhnisch 2012, S. 227).

Neben der Idee von Jugend als Freiraum bzw. Jugend als von bestimmten Aufgaben entpflichtende Lebensphase, die im Kontext des Moratoriumskonzepts diskutiert werden können, lassen sich im pädagogischen Fachdiskurs diverse Bezüge zur Bedeutung von Freiraumerleben und -erstreben ,im Alltag“ finden, auch wenn diese selten unter dem Begriff „Freiraum“ gefasst werden. So beschreibt beispielswei- 
se Lothar Böhnisch (2012) im Kontext der Lebensbewältigung, die Notwendigkeit gesellschaftlicher Integration für die Sozialisation, für die wiederum biografische Handlungsfähigkeit bedeutsam ist (Böhnisch 2012, S. 223). Die Gesellschaft kann ,aber nur dann auf einen flexiblen, optionsoffenen und damit integrationsfähigen Menschen [...] [hoffen], wenn sie Räume zulässt, in denen er zu sich kommen und bei sich selbst sein kann“ (Böhnisch 2012, S. 227), sich also als handlungsfähig erlebt. Implizit lässt sich hier die Relevanz von Freiräumen erkennen, in denen Menschen sich mit sich selbst auseinandersetzen und eine Balance zwischen gesellschaftlichen Erwartungen und sich herstellen können (Böhnisch 2012, S. 221). Daran anschlussfähig, aber stärker auf das Kindes- und Jugendalter bezogen, sind Heiner Keupps (2003) Arbeiten zur Identität. Auch er geht davon aus, dass „Identitätsarbeit [...] als Bedingung und als Ziel die Schaffung von Lebenskohärenz [hat]“ (Keupp 2003, S. 10). Hierfür benötigen Kinder und Jugendliche - so betont er explizit „Freiräume, um sich selbst zu entwerfen und gestaltend auf ihren Alltag einwirken zu können“ (Keupp 2003, S. 10). Vor dem Hintergrund pädagogischer Betrachtungen ist dann zu berücksichtigen, dass Freiräumen, wenn sie in Zusammenhang mit institutionellen pädagogischen Kontexten stehen, Vorstrukturierungen und Pädagogisierungen inhärent sind und damit auch eine Funktionalisierung des Freiraums einhergeht. Wenn Freiräume also ,als Räume, die - teilweise innerinstitutionell, zu Teilen am Rand, zu Teilen aber auch außerhalb der institutionellen Strukturen jungen Menschen eine Entfaltung ihrer Jugendlichkeit jenseits des Überlieferten ermöglichen“ (Deutscher Bundestag 2017, S. 109), gefasst werden, dann sind immer auch die Grenzen entsprechender Freiräume mit zu beobachten.

Gleichzeitig bleibt eher diffus, was „Freiraum“ eigentlich meint. Obwohl davon ausgegangen wird, dass die Bedeutung von Freiräumen und ihre Gestalt abhängig von dem Kontext sind, in dem sie diskutiert werden (Freiräume bedeuten so in der offenen Jugendarbeit anderes als in Schule oder Familie), wird folgend ein abstrakter Vorschlag zur Beschreibung von Freiraumerleben unterbreitet, von dem ausgegangen wird, dass dieser handlungsfeldübergreifende Gültigkeit besitzt.

\subsection{Freiraum - ein theoretischer Bestimmungsversuch}

Dem „Freiraum“ liegen mit dem ersten Begriffsbestandteil „frei“ vielfältige Attribute zu Grunde: „frei“ kann entsprechend ohne Einschränkungen, unabhängig von anderen, von bestimmten Aufgaben entbunden und nicht eingesperrt bzw. nicht kontrolliert meinen. In diesen Verständnissen bilden sich letztlich verschiedene Dimensionen wie Raum und Zeit, aber auch inhaltliche Vorstellungen darüber ab, welche Funktionen Freiräume erfüllen können bzw. was Freiraum ermöglichen soll. Entsprechend kann der zweite Wortbestandteil „Raum“ auch zu Fehlinterpretationen führen, da Freiräume nicht ausschließlich über freie Räume erlebbar sind.

Als konstitutiv für Freiräume kann beispielsweise mit J. Zinneckers (2000) Moratorium die Entlastung von ausgewählten Aktivitäten und von Verantwortung in einer bestimmten Alters- bzw. Zeitspanne, verbunden mit der Möglichkeit zu experimentieren, betrachtet werden (Zinnecker 2000, S. 38). Entsprechend können Freiräume wahlweise als Experimentierräume, als Spielräume, kontrollfreie Räume, Gegenkulturen oder Räume zur eigenen Gestaltung verstanden werden. Die AGJ 
(2016) hebt hervor, dass sich Freiräume vor allem durch freie Zeiten konstituieren, die Jugendliche selbstständig gestalten, dies aber von den jeweiligen Orten und damit auch Räumen (z.B. öffentliche Räume, spezifische Jugendräume) abhängig sind (AGJ 2016, S. 5 f.). Die Dimension des Raumes werden u.a. von Ahmet Derecik (2012) und Florian Glowatz-Frei (2015) im Kontext von Freiräumen in der (Ganztags-)Schule als auch vom Bundesministerium für Verkehr, Bau und Stadtentwicklung (BMVBS) (2010) in den Fokus gerückt. Letztere betonen, dass Freiräume „Spiel-, Erlebnis- und Aufenthaltsräume, Orte der Bewegung und Begegnung sowie Orte des eigenständigen Lernens" (BMVBS 2010, S. 3) darstellen.

Im Folgenden wird entsprechend davon ausgegangen, dass Freiräume keine konkreten Zeiten oder Räume darstellen, sondern dass das Erleben von Freiräumen von der Wahrnehmung verfügbarer Zeiten und Räume maßgeblich beeinflusst wird. Freiraum ist, so hier die Annahme, vom subjektiven Empfinden abhängig, das wiederum von individuellen, interaktionalen wie institutionellen Aspekten beeinflusst wird. Als zentral für das Erleben von Freiraum werden im Folgenden die drei Dimensionen freie Zeit, rückzugs- bzw. kontrollarme Räume sowie Selbstbestimmungserleben vorgeschlagen.

\subsubsection{Dimension 1: Freie Zeit}

Dass bei einer Betrachtung von Freiräumen eine zeitliche Dimension berücksichtigt werden sollte, lässt sich aus Synonymen wie „Auszeiten“ (Deutscher Bundestag 2017, S. 22) ableiten. Für die Auseinandersetzung mit Zeit und der Beschreibung ihrer Bedeutung für das Erleben von Freiraum ist eine Differenzierung in subjektive und objektive Zeit, die Michael Görtler (2016) bezugnehmend auf Albert Einstein und Henri Bergson vornimmt, zentral (Görtler 2016, S. 19). Dabei meint die objektive Zeit die alle Menschen gleichermaßen betreffenden Zeitkategorien. Hingegen umfasst die subjektive Zeit die individuellen Zeitwahrnehmungen und -bewertungen (vgl. Görtler 2016). Damit einher geht die strukturierende Funktion, die Zeit ausübt und die individuell, aber auch aus der Umwelt heraus, als Rahmung von Handlungen, Aufgaben etc. gesehen werden kann (Deußer und Nebelin 2009, S. 9). Eine solche ordnende Funktion liegt beispielsweise der Trennung von Freizeit und Arbeit zu Grunde, sofern Freizeit nicht nur als Gegenbegriff zur Arbeit, sondern als frei verfügbare Zeit definiert wird (Opaschowski 2008, S. 315). Freie Zeiten unterliegen dabei schon von jeher gesellschaftlichen Zeitregimen, in denen die Verwendung von Zeit bestimmten Regeln unterworfen ist (Prahl 2015, S. 4). Mit frei verfügbaren Zeiten bzw. Freizeit ist daher auch ein Gewinn an Handlungsspielräumen verbunden, auch wenn die darin liegenden Freiheitsgrade nicht beliebig sind und sozial kontrolliert werden (Opaschowski 1994, S. 442f.). Insofern ist Freizeit als ,relativ frei wählbare und selbstbestimmbare Lebenszeit" (Opaschowski 2008, S. 315) kein Synonym für Freiraum, sondern eine mögliche Voraussetzung selbstbestimmter und freiwilliger Aktivität (Opaschowski 1994, S. 443).

Zentral ist daher, dass Zeiten einerseits durch z. B. Institutionen oder die Gesellschaft auf der objektiven Ebene das Leben von Individuen und Gruppen beeinflussen und damit Freiräume ermöglichen oder behindern können. Subjektiv wird diese objektive Zeit unterschiedlich erlebt. Insofern lässt sich nicht eindeutig klären, 
inwieweit freie Zeiten von Individuen auch gleichzeitig als Freiräume eingeschätzt werden, da dies wiederum von den weiteren Dimensionen abhängig ist.

\subsubsection{Dimension 2: Raum}

Nimmt man stärker die räumliche Perspektive der Freiräume in den Blick, so geht mit dieser eher die Frage nach Rückzugs- und Erprobungsmöglichkeiten (Deutscher Bundestag 2017, S. 22) einher. Der hier vorgestellt Ansatz folgt der Logik der relationalen Räume und geht damit über territorial und von Menschen unabhängig vorhandenen Raumlogiken hinaus (Löw 2001, S. 264). Räume entstehen demnach nicht unabhängig von sozialen Gütern, Menschen und Interaktionen. Daraus resultiert, dass an einem territorial festgelegten Ort mehrere Räume existieren können (Löw 2001, S. 271) und damit auch in territorial unbestimmten Räumen relationale Räume vorhanden sind. Entsprechend ist die Räumen oft zugeschriebene Zweckgebundenheit durchaus auch eine Frage pädagogisch ge- und verplanter Räume.

Werden Räume als Freiräume betrachtet, so sollten mindestens zwei verschiedene „Räume“ berücksichtigt werden, die sich mit den Polen öffentlich und privat verknüpfen lassen. Im Kontext des öffentlichen Raums liegt der Fokus auf Experimentierräumen und auf der bereits angedeuteten Zweckentfremdung und Aneignung von Räumen, die auch mit Eigentätigkeiten bzw. „Situations- und Raumveränderung durch Kinder und Jugendliche“" (Deinet 2005, S. 51) einhergehen. Andererseits werden im öffentlichen Raum Handlungsspielräume und Freiräume limitiert, da hier Grenzen hergestellt werden und so beispielsweise der Bewegungsraum eingegrenzt wird (Deinet 2005, S. $46 \mathrm{ff}$.).

Bei Räumen, deren Funktion die Bereitstellung von Rückzugsmöglichkeiten ist, geht dies insbesondere mit dem Konzept der lokalen Privatheit bzw. Privatsphäre einher. Privatheit kann als notwendige Bedingung für Autonomie betrachtet werden bzw. die eigene Identität schützen und ermöglichen (Rössler 2017, S. $281 \mathrm{ff}$; Trescher 2015, S. 138). Dabei wird Privatsphäre als Teil der Privatheit durch einen räumlich-sozialen Rückzugsort hergestellt und ermöglicht, zu dem die Öffentlichkeit nicht ungefragt Zugang hat (Trescher 2015, S. 138f.). So wird das eigene Zimmer im Kindes- und Jugendalter im Jugendbericht als Freiraum-Element hervorgehoben (Deutscher Bundestag 2017, S. 111). Mit der nicht-ungefragten Zugänglichkeit geht vor allem die Chance einher, individuelle Verhaltensweisen unabhängig von anderen auszudrücken und den Raum selbst räumlich zu inszenieren (Rössler 2001, S. 256f.). „Sich den Blicken anderer entziehen zu können, ist offenbar für das Gelingen von Autonomie elementar: wenn ein Subjekt die Möglichkeit hat, unbehelligt von anderen mit sich selbst allein zu sein, wenn es dies wünscht, so trägt dies sicherlich entscheidend dazu bei, dass es zu Formen gelingender Selbstbestimmung in der Lage ist, dass es sich klar werden kann darüber, was es denkt, will, wie es sein und leben will“" (Rössler 2001, S. 274).

\subsubsection{Dimension 3: Selbstbestimmtheit}

Da Freiraum weder allein einen bestimmten Raum oder eine bestimmte Zeit darstellt, noch ausgewählte Aktivitäten umfasst, sollte eine dritte Dimension „Selbstbestimmt- 
heit" zur Beschreibung von Freiräumen hinzugefügt werden. Selbstbestimmung allgemein meint ,die Bestimmung des Menschen über das eigene Wollen, Werden und Tun“ (Tenorth und Tippelt 2012, S. 649), womit auch auf die Machtausübung über sich selbst verwiesen wird (Waldschmidt 2012, S. 20). Selbstbestimmung wird hier vor allem als „Selbstgestaltung“ des eigenen Lebens verstanden, die Anne Waldschmidt (2012) von „Selbstbeherrschung“, „Selbstinstrumentalisierung“ und „Selbstthematisierung“ als Deutungen von Selbstbestimmung differenziert (Waldschmidt 2012, S. 70). Selbstbestimmung als Selbstgestaltung geht mit der Frage: „Wie will ich leben?“ (Waldschmidt 2012), einher. Dabei kann Selbstbestimmung als notwendige Bedingung für ein als sinnvoll erlebtes Leben eingeordnet werden (Rössler 2017, S. 31).

Zur Beschreibung eines Selbstbestimmungserlebens bzw. selbstbestimmter Handlungen ist das Kontinuum zwischen Kontrolle und Zwang auf der einen und der freien Wahl auf der anderen Seite ausschlaggebend (Deci und Ryan 1993, S. 225). Dementsprechend kann das Selbstbestimmungserleben durch Eingriffe, Erwartungen etc. von außen beschränkt werden. Allerdings können Menschen sich durchaus als selbstbestimmt erleben, selbst wenn sie von außen determiniert werden (Prozess der Internalisation und Integration) (Deci und Ryan 1993, S. 227). Zu fragen ist sogar, ob wirkliche Selbstbestimmung bzw. Autonomie möglich ist, da ,autonomieerschwerdene oder -verhindernde Strukturen [...] zu unserem autonom gelebten Alltag [gehören]“ (Rössler 2017, S. 21).

Entsprechend ist das Erleben von Freiräumen besonders vom Gefühl der Selbstbestimmung abhängig und dadurch ein individuelles Empfinden, das jedoch durch institutionelle Strukturen beeinflusst wird. Ein Dilemma besteht hier einerseits in der Vorstellung, Selbstbestimmung in (nicht freiwillig besuchten) Organisationen (z.B. Schule, Internat) zu ermöglichen und zu erleben (Katenbrink 2014). Daneben stellt sich andererseits die Frage nach der Fähigkeit zur Selbstbestimmung - die, nimmt man beispielsweise Bezug auf Entwicklungsaufgaben (u. a. Hurrelmann und Quenzel 2016, S. 33 f.), sich in der Jugendphase erst herausbilden soll. Insofern liegt hier eine Ambivalenz zwischen der Forderung nach Selbstbestimmungsmöglichkeiten bei gleichzeitiger Beschränkung dieser aufgrund der Vorstellung einer sich noch entwickelnden Fähigkeit vor, die auch das Ermöglichen von Freiräumen beeinflusst.

\section{Freiräume im Internat}

Eine Betrachtung des Freiraumerlebens in Internaten bietet aufgrund ihres besonderen, da allumfassenden Charakters - der auch mit Erving Goffman (1981) als ,totale Institution“ beschrieben werden kann (Kalthoff 1997) - eine Chance zur Untersuchung der verschiedenen Freiraum-Dimensionen und weiteren Einflüssen. Da im Zentrum der empirischen Studie der außerschulische Kontext, also organisationale, den Alltag rahmende Elemente vor und nach der Schule sowie Möglichkeiten der Selbstpositionierung und Einflussnahme auf den außerschulischen Alltag, liegt, sollen im Folgenden einige bedeutende Merkmale von und Forschungsergebnisse zu Internaten, die allerdings aus Studien stammen, die nicht explizit das Freiraumerle- 
ben untersucht haben, und den strukturell vergleichbaren Feldern der Ganztagsschule und stationären Erziehungshilfe kursorisch präsentiert werden.

Insbesondere die räumliche Verdichtung auf das Internatsgelände sowie die zeitlich rhythmisierte Tagesstruktur (Haep 2015, S. $128 \mathrm{f}$.) knüpfen an die eben eingeführten Dimensionen des Freiraumerlebens an. Verwirklicht werden diese durch diverse Regeln, Aufgaben und Erwartungen, die an die Jugendlichen gerichtet sind und deren Einhaltung sowohl durch das Personal als auch Peers, die als zentrale Bezugspersonen anzusehen sind (Gibson 2017, S. 440f.), kontrolliert werden. Sowohl Herbert Kalthoff (1997) als auch Anja Gibson (2017) und Katrin Peyerl (2020) beschreiben, dass Regelkataloge nicht nur der Alltagsorganisation, sondern auch der Kontrolle der Schüler*innen dienen, womit Einschränkungen ihrer Handlungsspielräume einhergehen, auch wenn für Jugendliche, so Nora Katenbrink (2014), diese Regeln unterschiedliche Bedeutung besitzen und Praktiken zur Folge haben (z.B. Schutzfunktion und Akzeptanz vs. Kontrollerleben und Regelüberschreitung) (Katenbrink 2014, S. 100 ff.). Ähnliches ist aus der Forschung zur stationären Erziehungshilfe bekannt. So erläutert u. a. Heinz Abels (2006), dass Regeln und Strukturen charakteristisch sind, die die Jugendlichen allerdings nicht selbst aushandeln können (Abels 2006, S. 96). K. Peyerl (2020) stellt außerdem einen Zusammenhang zwischen dem Wunsch nach Einflussnahme auf das eigene Leben sowie das Internat und der erlebten Wirksamkeit in organisierten Formen von Mitbestimmung für die Praktiken im Umgang mit Regeln und Kontrollen bzw. dem Grad der Anpassung an organisationale Konformitätserwartungen durch die Bewohner*innen heraus (Peyerl 2020, S. 289 ff.). Daneben beschreibt sie - wie N. Katenbrink (2014) - akzeptierende Praktiken, die aber eher auf eine Transitions- und Entwicklungsorientierung der Jugendlichen zurückgeführt werden können.

Die meist periodisch organisierte Zeiteinteilung erfüllt einerseits eine Kontrollfunktion (Kalthoff 1997), die das Freiraumerleben einschränken kann, andererseits soll diese auch der Förderung von Orientierung und Sicherheit im Internat dienen (vg. Gibson 2017, S. 319). Aus der Ganztagsschulforschung lässt sich mit FritzUlrich Kolbe, Kerstin Rabenstein und Sabine Reh (2006) beschreiben, dass „Jugendliche freie Zeit in der Schule teilweise als ,Leerlauf" empfinden“" Kolbe et al. 2006, S. 26), weil sie diese freien Zeiten - dazu zählen vor allem Freistunden, aber auch Pausen - für sich nicht sinnvoll nutzen können. Ihr selbstbestimmter Handlungsspielraum ist also, trotz freier Zeiten, eingeschränkt. Gleichzeitig stellen F.-U. Kolbe, K. Rabenstein und S. Reh (2006) dar, dass Jugendliche ab der zehnten Klasse weniger vorstrukturierte Angebote in den unterrichtsfreien Zeiten bevorzugen würden, damit sie die Zeit selbstbestimmt und abwechslungsreich gestalten können (Kolbe et al. 2006, S. 26ff.). Regina Soremski (2013) beschreibt im Kontext ihrer qualitativen Studie hingegen, dass trotz festgelegter Zeit- und Raumstrukturen im Freizeitbereich der Ganztagsschule Autonomie-Spielräume für Jugendliche vorhanden sind und von diesen wahrgenommen werden (vgl. auch Derecik 2012).

Hinsichtlich der räumlichen Gegebenheiten ist neben der Tatsache, dass sich der Alltag der Jugendlichen überwiegend auf dem Internatsgelände vollzieht, insbesondere auf fehlende Rückzugsmöglichkeiten hinzuweisen, wie A. Gibson (2017) in ihrer Studie herausgearbeitet hat. Zurückführen lässt sich dies auf die teils fehlenden Einzelzimmer, die Privatsphäre ermöglichen könnten, es wird aber auch von der 
interaktionalen Ebene, also dem Verhältnis und Umgang mit Peers und Angestellten beeinflusst. In der Ganztagsschulforschung wird vor allem ,ein dringendes Bedürfnis nach durch keinerlei pädagogisches Personal kontrollierten Räumen“ (Kolbe et al. 2006, S. 29) dargestellt, während in der Internatsforschung der besondere Einfluss der Peers stärker hervorgehoben wird. A. Gibson arbeitet (2017) beispielsweise den normierenden Einfluss dieser und damit verbundene Kohärenzbildungsprozesse heraus, die Anpassungserfordernisse zur Folge haben. Hierdurch ,wird man zu einem Teil der Gemeinschaft und erfährt innerhalb des internatsschulischen Raumes Schutz und Halt und kann auf Raum- und Zeitstrukturen zur individuellen Entfaltung der Talente und Begabungen zurückgreifen“ (Gibson 2017, S. 409). Ähnliches schildern auch Werner Helsper, Jeanette Böhme, Rolf-Torsten Kramer und Angelika Lingkost (2001), die u. a. beschreiben, dass Jugendliche nur dann partizipieren und autonom handeln können, wenn sie nicht entgegen der schulischen Ordnung handeln, was wiederum das Erleben von Selbstbestimmung beeinflusst (Helsper et al. 2001, S. 575).

\section{Zwischenbilanz und empirische Fragestellung}

Die postulierte Dimensionierung von Freiraum findet durchaus Entsprechungen in den zusammengetragenen Forschungsergebnissen, welche auch Hinweise auf Einflüsse auf die Wahrnehmung von Freiräumen von Internatsbewohner*innen geben. So zeigen sich in den Studien Internatsstrukturen, die Freiräume ermöglichen oder behindern, sowie Praktiken in Internaten, die eher als Anpassung an oder als Auflehnen gegen solche Strukturen gedeutet werden können. Deutlich wird ein Wechselspiel zwischen Regeln und Strukturen einerseits und ihrer Aushandlung bzw. den informellen Umgangspraktiken Jugendlicher andererseits.

Diese Befunde werden im Folgenden genutzt, um das vorgeschlagene theoretische Freiraummodell auf seine empirische „Bewährung“ zu prüfen. So wird analysiert, inwieweit die Wahrnehmung eigenen Freiraums sich über die Wahrnehmung von Rückzugsräumen, Wahrnehmung freier Zeiten und der eigenen Selbstbestimmungsmöglichkeiten erklären lässt. Da, wie gezeigt, diese Wahrnehmung nicht losgelöst von institutionellen, interaktionalen sowie personalen Faktoren betrachtet werden kann, wird ein Modell geprüft, das alle diese Ebenen umfasst. Angenommen wird dabei insbesondere der Einfluss der Beziehungen zu Betreuer*innen, die u.a. Freiräume unterhalb formaler Regelungen gewähren können, und Mitbewohner*innen, mit denen gemeinsame Werteorientierungen und kollektive Wahrnehmungen ausgeprägt werden (u.a. Gibson 2017, S. 408). Außerdem werden mit zunehmender Klassenstufe mehr Freiräume von den Internaten gewährt, beispielsweise ein eigenes Zimmer, so dass auch für die Klassenstufe ein bedeutsamer Effekt vermutet wird. 
Tab. 1 Stichprobe der befragten Internatsschüler*innen

\begin{tabular}{ll}
\hline & $\begin{array}{l}\text { Internatsschüler*innen } \\
(n=117)\end{array}$ \\
\hline Mädchenanteil & $36 \%$ \\
Klassenstufe 7 & $9 \%$ \\
Klassenstufe 9 & $34 \%$ \\
Klassenstufe 11 & $57 \%$ \\
Anteil mit mind. einem Elternteil mit Migrationshintergrund & $44 \%$ \\
Anteil der Befragten mit Geschwistern & $87 \%$ \\
Sozioökonomischer Status der Eltern (HISEI, Anteil aus oberem Drit- & $86 \%$ \\
tel der Gesamtbev.) & \\
Elternteil Akademiker*in & $88 \%$ \\
Anteil zusammenlebender Eltern & $66 \%$ \\
Durchschnittsalter in Jahren & 16,6 (MW); 1,7 (SD) \\
Durchschn. bisherige Zeit im Internat in Monaten & 23,3 (MW); 13,7 (SD) \\
\hline
\end{tabular}

\section{Methodik}

Die empirischen Analysen erfolgen über die explorative quantitative Studie „Aufwachsen im Internat", in welcher in fünf Internatsschulen (aus zwei Internatsverbänden) Internatsbewohner*innen und externe Schüler*innen derselben Schulen befragt wurden (Züchner und Peyerl 2018). Die Einbeziehung der Internate hing von deren Bereitschaft ab, so dass nicht automatisch davon ausgegangen werden kann, dass die hier erhobene Stichprobe stellvertretend für alle altersgleichen Internatsbewohner*innen in den beiden Verbänden ist. So sind die im Folgenden verwendeten Signifikanzangaben mit Vorsicht zu genießen, inhaltlich werden vor allem Effektstärken interpretiert. Die Erhebungen fanden im Juli/September 2016 mittels Fragebögen vor Ort statt. Um vergleichbare Altersgruppen zu gewährleisten, wurden vor den Sommerferien ausschließlich Jugendliche der siebten, neunten und elften Klassen, nach den Sommerferien der achten, zehnten und zwölften Klassen beteiligt ${ }^{12}$. Die externen Schüler*innen erhielten eine kürzere Version des Fragebogens. Insgesamt lagen zur Auswertung aus den fünf Internatsschulen Antworten von 117 internen und 227 externen Schüler*innen vor. Pro Internatsschule wurden zwischen 19 und 32 interne und 28-53 externe Schüler*innen erreicht. Die folgenden Analysen beziehen sich nur auf die Internatsbewohner*innen (vgl. Tab. 1).

\footnotetext{
${ }^{1}$ Die im Sommer nach Schulwiederbeginn befragten Jugendlichen der achten, zehnten und zwölften Klasse wurden der jeweils unterhalb liegenden Klassenstufe für die Auswertung zugeordnet, da diese einer Jahrgangsgruppe angehören.

2 Chin (1998) geht bei einer standardisierten Pfadkoeffizienten von $\geq \pm 0,2$ von bedeutsamen Effekten aus. Daher sind hier und im Folgenden in den Pfadmodellen standardisierte Koeffizienten in diesem Bereich abgetragen.
} 


\subsection{Untersuchungsansatz für die Fragestellung}

Die Operationalisierung der Fragestellung erfolgt über ein Pfadmodell, das zunächst untersucht, inwieweit sich das über die drei Dimensionen „Zeit“, „Raum“ und „Selbstbestimmung“ konzipierte Modell zum Freiraumempfinden auch empirisch abbilden lässt. Darüber hinaus wird davon ausgegangen, dass - möglicherweise vermittelt über diese Dimensionen - institutionelle, interaktionale und personale Faktoren einen Einfluss haben. In der Annahme, dass das Internat als Lebensort der Jugendlichen das Erleben von Freiräumen maßgeblich bedingt - u. a. aufgrund der verschiedenen Internatskonzeptionen - sollten Freiräume zwischen den Internaten unterschiedlich empfunden werden (= institutionelle Ebene). Damit verbunden ist die interaktionale Ebene: Ausgegangen wird davon, dass das Erleben eigener Freiräume und Selbstbestimmung nicht nur von formalen Regeln und Konzeptionen der Internate, sondern auch vom Personal (die im Zweifelsfall diese formalen Regeln der Institutionen verhandeln oder durchsetzen) und Mitbewohner*innen (mit eigenen Vorstellungen) abhängen. Auf der personalen Ebene wird insbesondere ein Einfluss von Alter und Klassenstufe angenommen, u. a. da vermutet wird, dass mit zunehmendem Alter das Freiraumbedürfnis steigt und gleichzeitig mehr Privilegien mit steigender Klassenstufe gewährt werden (u. a. eigenes Zimmer, Ausgehzeiten). Ergänzend werden als Kontrollvariablen weitere soziodemographische Merkmale aufgenommen (Geschlecht, Bildungsstand der Eltern, Migrationshintergrund). Die Operationalisierung zentraler Variablen erfolgt wie folgend:

- Freiraum-Empfinden: Das Freiraum-Empfinden im Internat wurde durch ein Item erhoben. Dabei sollten die Jugendlichen auf einer Skala von 1 (kein) bis 10 (viel Freiraum) ankreuzen: „Wie viel Freiraum, findest du, hast du im Internat“ (MW: 6,25; SD: 1,$94 ; n=116$ ). Sechs Prozent der Varianz der Variable entfällt auf den Kontext Internat.

- Selbstbestimmung: Zu Erfassung des Erlebens von Selbstbestimmung wurde eine Skala $^{3}$ über eine Hauptachsenanalyse mit insgesamt neun Items gewonnen, bestehend aus sechs vierstufigen (von $1=$ trifft gar nicht zu bis $4=$ trifft sehr $\mathrm{zu}$ ) Items, z. B.: „Ich kann bestimmen, wie mein Zimmer aussieht“; ,Ich kann selbst entscheiden, wann ich aufstehe und ins Bett gehe" (MW: 2,49; SD: 0,59; $n=117$ ). $32 \%$ der Varianz entfällt auf den Kontext Internat.

- Freie Zeit: Die Zeit-Dimension wird durch eine skalierte Variable (1= „trifft gar nicht zu“ bis $4=$,,trifft sehr zu“) erfasst: „Ich hätte gerne mehr freie Zeit im Internat" (MW: 2,98; SD: 0,84; $n=114$ ). Der Varianzanteil auf Internatsebene beträgt acht Prozent.

- Freie „(Rückszugs)Räume“: Die Dimension des Raumes wird durch die Frage nach Rückzugsräumen - und damit nach Privatsphäre - in einer skalierten Variable ( $1=$,,trifft gar nicht zu“ bis $4=$,,trifft sehr zu“) erfasst: „Ich hätte gerne mehr Räume, in denen man ungestört sein kann“ (MW: 2,80; SD: 0,96; $n=116$ ). Der Varianzanteil auf Internatsebene beträgt elf Prozent.

\footnotetext{
${ }^{3}$ Die eingesetzte Skala „Selbstbestimmungserleben“ wurde über die Mittelwerte der Items gebildet mit einem Cronbachs Alpha 0,70.
} 
Weitere unabhängige Variablen lassen sich in institutionelle, interaktionale und personenbezogene Variablen untergliedern und wurden wie im Folgenden dargestellt erhoben:

- Institutionelle Ebene: Internatszugehörigkeit: Entsprechend der fünf im Sample untersuchten Internate, erhielt jede*r Jugendliche für seine Internatszugehörigkeit eine Ausprägung von 1-5 auf einer nominalen Variable zum besuchten Internat. Wie bereits bei der Darstellung des Samples vorgestellt, wurden im arithmetischen Mittel 23 Internatsbewohner*innen je Internat befragt (I1: 32; I2: 20, I3: 19, I4: 22; I5: 23).

- Interaktionale Ebene: Zum einen wurde eine Skala ${ }^{4}$ „Verhältnis zu Betreuer*innen " aus sieben Items gebildet (Beispielitems: „Ich werde ernst genommen“; „Ich habe ein vertrauensvolles Verhältnis zu meinen Betreuer*innen“ [MW: 3,11; SD: 0,$63 ; n=117])$. Auch eingesetzt wurde eine Skala ${ }^{5}$,Verhältnis zu Mitbewohner*innen“, die aus fünf Items entwickelt wurde (Beispielitems: „Ich bin gern mit ihnen zusammen“. „Ich habe ein vertrauensvolles Verhältnis zu den anderen Internatsbewohner*innen" [MW: 3,17; SD: 0,68, $n=113]$ ).

- Personale Ebene: Diese beinhaltet die personenspezifischen Daten zu Geschlecht (binär codiert), Migrationshintergrund (binär codiert, Zuwanderung mindestens eines Elternteils), akademischem Elternhaus (binär codiert, mind. ein Elternteil mit akadem. Abschluss), dem Alter der Befragten, die besuchte Klassenstufe sowie die bisherige Wohndauer im Internat, welche metrisch erfasst wurden.

\subsection{Analysemethoden}

Die Analysen bauen auf einem Pfadmodell, das zunächst als einfaches Pfadmodell mit einer zu erklärenden Variable und drei bedingenden Indikatoren für Raum, Freie Zeit und Selbstbestimmung geschätzt wird, auf. Das Pfadmodell wurde gewählt, da von korrelativen Beziehungen und von direkten sowie indirekten Effekten der Variablen ausgegangen wurde, die mit einem Pfadmodell modelliert werden können. Die getestete Struktur der Daten (Personen in Internaten) wurde in den Pfadmodellen durch die Verwendung der Standardfehlerkorrektur der Software Mplus berücksichtigt. In einem zweiten Schritt wurde das Pfadmodell um institutionelle, interaktionale und individuelle Einflussfaktoren erweitert. Mit Blick auf die Kontrolle des Einflusses der einzelnen Internate wurde das Pfadmodell als Regressionsmodell mit festen Effekten geschätzt, in dem vier der fünf Internate als Dummyvariablen aufgenommen wurden und somit die unerklärte Varianz weiterer über die benannten institutionellen Einflüsse auf sich ziehen (vgl. dazu Langer 2009), ein Mehrebenenmodell mit Zufallseffekten war wegen der geringen Fallzahl auf Ebene 2 (= 5 Internate) nicht sinnvoll (vgl. dazu Maas und Hox 2005, S. 90).

${ }^{4}$ Cronbachs Alpha $=0,88$.

5 Cronbachs Alpha =0,87. 
Abb. 1 Pfadmodell zur ,Erklärung" von Freiraumerleben $(n=110)$ (Standardisierte Koeffizienten [Standardfehler in Klammern])

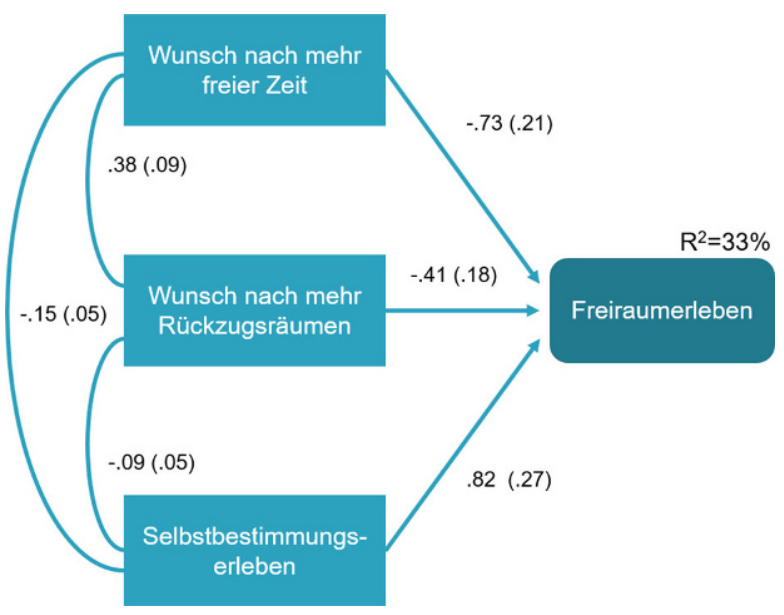

\section{Ergebnisse}

\section{1 Überprüfung des theoretischen Modells zum Freiraum-Erleben}

In einem ersten Schritt wird das theoretisch formulierte Modell zur Konstitution von Freiraumerleben anhand der Studiendaten über ein einfaches Pfadmodell geschätzt (siehe Abb. 1). Ausgangspunkt war die These, dass Freiraumerleben von dem Wunsch nach mehr freie Zeit, den erlebten Rückzugsmöglichkeiten sowie den wahrgenommenen Selbstbestimmungsmöglichkeiten bedingt wird, dass letztere Variablen aber auch untereinander in Beziehung stehen.

Das Pfadmodell zeigt bedeutsame Einflüsse der einbezogenen Variablen auf das Freiraumerleben ${ }^{6}$. Zudem bildet sich eine signifikante Korrelation zwischen dem Wunsch nach mehr freier Zeit und dem Selbstbestimmungserleben ab - bei einer Varianzaufklärung von $33 \%$.

\subsection{Institutionelle, interaktionale und individuelle Einflüsse auf das Freiraumerleben}

Aufbauend auf das erste Modell stellt sich die Frage, welche Faktoren das Erleben von Freiräumen - und eben auch das Erleben von freier Zeit, räumlichen Möglichkeiten sowie Selbstbestimmung - im Internat beeinflussen und inwieweit die im ersten Modell angenommenen Zusammenhänge auch bei Ergänzung weiterer Prädiktoren bestätigt werden. Dazu wird zunächst die Annahme aufgegriffen, dass die Wahrnehmung von eigener Verfügung über Zeiten und Räume sowie Selbstbestimmung wesentlich durch den Rahmen der Internate (institutionelle Strukturen sowie auch Interaktionen) bedingt ist.

\footnotetext{
${ }^{6}$ Chin (1998) geht bei einer standardisierten Pfadkoeffizienten von $\geq \pm 0,2$ von bedeutsamen Effekten aus. Alle Koeffizienten sind signifikant mit $p<0,05$.
} 
Abb. 2 Mittelwert der empfundenen Freiräume im Internat nach Internaten $(n=116)$ $\left(\mathrm{F}=1,74 ; \mathrm{df}=4 ;\right.$ eta $^{2}=0,06 ;$ $p=$ n.s.)

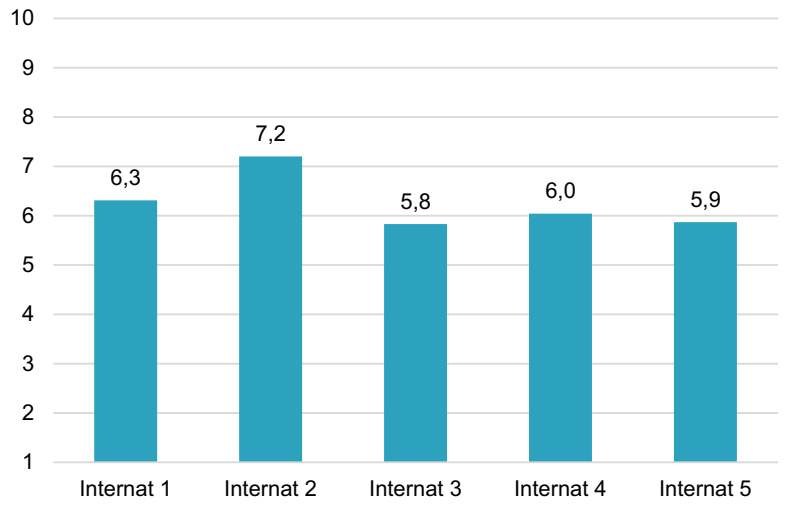

Der Blick auf die Mittelwerte zum empfundenen Freiraum zeigt, dass die Jugendlichen ihre Freiräume im Internat als eher durchschnittlich ausgeprägt einschätzen $(M W=6,25)$; mit einem eta ${ }^{2}$ von 0,06 findet sich ein nach Cohen (1988) mittlerer Effekt für den Unterschied zwischen den Institutionen (vgl. Abb. 2) ${ }^{7}$.

Mittlere Unterschiede finden sich zwischen den Internaten auch für die Selbstbestimmungsskala $\left(\mathrm{F}=13,02 ; \mathrm{df}=4\right.$; eta $\left.^{2}=0,32 ; p<0,05\right)$, für den Wunsch nach mehr freie Zeit $\left(\mathrm{F}=3,284 ; \mathrm{df}=4\right.$; eta $\left.^{2}=0,11 ; p<0,05\right)$ und kleinere für den Wunsch nach mehr Rückzugsmöglichkeiten $\left(\mathrm{F}=2,258 ; \mathrm{df}=4\right.$; eta ${ }^{2}=0,07 ; p=\mathrm{n} . \mathrm{s}$.). Diese heterogenen Befunde deuten darauf hin, dass das Freiraumerleben nicht nur von institutionellen Rahmungen, sondern auch von interaktionalen und personenbezogenen Einflüssen abhängig ist. Um einen Überblick über die Zusammenhänge zu erhalten, wurden die skizzierten Variablen zunächst miteinander korreliert (vgl. Tab. 2).

Über die Korrelationen zwischen den im ersten Pfadmodell aufgenommenen Variablen hinaus zeigen sich jedoch nur noch bedingt bedeutsame Zusammenhänge: Interessant sind die Korrelationen von Alter und Klassenstufe mit anderen Variablen. Mit steigender Klassenstufe steigt auch der empfundene Freiraum, auch steigt mit der Klassenstufe die empfundene Selbstbestimmung und wird der Wunsch nach mehr freier Zeit seltener (hier greifen möglicherweise mit der Klassenstufe wachsende Privilegien), während mit höherem Alter das Selbstbestimmungsempfinden sinkt. Um nun die Einflüsse der institutionellen, interaktionalen und individuellen Faktoren auf das Freiraumempfinden zu untersuchen und dabei mögliche indirekte Effekte aufzudecken, wurde das Pfadmodell um diese benannten Variablen erweitert (vgl. Abb. 3).

Sichtbar wird zunächst, dass der angenommene Zusammenhang von Freiraum mit den drei Dimensionen erhalten bleibt. Die erklärte Varianz steigt gegenüber

\footnotetext{
7 Im Vergleich schätzen die die Jugendlichen ihre Freiräume im Internat als eher durchschnittlich ausgeprägt ein, während dieser in einer vergleichbaren Variable zur Familie eher hoch eingestuft wird (MW: 8,22; SD: 1,77; $n=115$ ). Dabei besteht keine bedeutsame Korrelation zwischen dem Freiraumempfinden in Familie und Internat $(r=0,08)$, was als Hinweis für die strukturelle Trennung der Orte im Alltag gedeutet werden kann.
} 
Tab. 2 Korrelationsmatrix der ausgewählten Variablen $(n=114)(*=p<0,05)$

\begin{tabular}{|c|c|c|c|c|}
\hline & $\begin{array}{l}\text { Empfundener } \\
\text { Freiraum im } \\
\text { Internat }\end{array}$ & $\begin{array}{l}\text { Wunsch nach } \\
\text { mehr freier } \\
\text { Zeit }\end{array}$ & $\begin{array}{l}\text { Wunsch nach } \\
\text { mehr Rück- } \\
\text { zugsräumen }\end{array}$ & $\begin{array}{l}\text { [S] Selbstbestim- } \\
\text { mungsmögl. im } \\
\text { Internat }\end{array}$ \\
\hline $\begin{array}{l}\text { Empfundener Frei- } \\
\text { raum im Internat }\end{array}$ & 1 & - & - & - \\
\hline $\begin{array}{l}\text { Wunsch nach mehr } \\
\text { freier Zeit }\end{array}$ & $-0,480^{*}$ & 1 & - & - \\
\hline $\begin{array}{l}\text { Wunsch nach mehr } \\
\text { Rückzugsräumen }\end{array}$ & $-0,394^{*}$ & $0,463 *$ & 1 & - \\
\hline $\begin{array}{l}\text { [S] Selbstbestim- } \\
\text { mungsmögl. im } \\
\text { Internat }\end{array}$ & $0,382 *$ & $-0,290 *$ & $-0,152$ & 1 \\
\hline $\begin{array}{l}{[\mathrm{S}] \text { Verhältnis zu }} \\
\text { Betreuer*innen }\end{array}$ & 0,324 & $-0,287$ & $-0,197 *$ & $0,290 *$ \\
\hline $\begin{array}{l}\text { [S] Verhältnis zu } \\
\text { Internatsbewoh- } \\
\text { ner*innen }\end{array}$ & 0,187 & $-0,095$ & $-0,165$ & 0,024 \\
\hline $\begin{array}{l}\text { Migrationsstatus der } \\
\text { Eltern }\end{array}$ & 0,058 & 0,017 & 0,091 & 0,111 \\
\hline $\begin{array}{l}\text { Eltern(teil) Akade- } \\
\text { mik. }\end{array}$ & 0,147 & 0,020 & $-0,051$ & 0,049 \\
\hline Alter (in Jahren) & $0,187 *$ & 0,151 & 0,070 & $-0,256^{*}$ \\
\hline $\begin{array}{l}\text { Zeit im Internat (in } \\
\text { Monaten) }\end{array}$ & 0,112 & $-0,106$ & $-0,003$ & $-0,050$ \\
\hline Klassenstufe & $0,184 *$ & $-0,246^{*}$ & $-0,068$ & $0,185^{*}$ \\
\hline $\begin{array}{l}\text { Weibliches Ge- } \\
\text { schlecht }\end{array}$ & $-0,196^{*}$ & 0,002 & 0,096 & $-0,103$ \\
\hline
\end{tabular}

dem vorigen Modell nur leicht an und die nun einbezogenen Variablen haben vor allem indirekte, über die drei Dimensionen vermittelte Effekte.

Auf individueller Ebene geht eine steigende Höhe der Klassenstufe, wie zuvor aufgrund der im Internat hiermit steigenden „Privilegien“ und Rechte (z. B. Ausgangszeiten) angenommen, mit mehr wahrgenommenen Selbstbestimmungsmöglichkeiten und einem geringeren Wunsch nach freier Zeit einher. Eine gute Beziehung zu den Betreuer*innen hat jeweils vergleichbare Einflüsse auf diese beiden Dimensionen, die sich mit einer gewissen Verhandelbarkeit von Möglichkeiten interpretieren lässt. Für die Beziehung zu den Mitbewohner*innen kann, anders als erwartet, weder ein direkter noch ein indirekter Einfluss nachgewiesen werden.

Über diese Faktoren hinaus zeigt sich die Bedeutung des jeweiligen Internatskontextes, der sich in jeweils drei Internaten (gegenüber dem Referenzinternat 5) als bedeutsam für den Wunsch nach mehr freier Zeit und auf das Selbstbestimmungserleben erweist ${ }^{8}$. Für das Freiraumempfinden haben die Internatskontexte ebenfalls eine indirekte, vor allem über diese beiden Dimensionen vermittelte Bedeutung.

\footnotetext{
${ }^{8}$ Im Modell deutet sich zudem über die beschriebenen Effekte ein eigener Einfluss der reformpädagogischen Ausrichtung von Internaten auf das Selbstbestimmungsempfinden an (Internat 2 und Internat 3).
} 


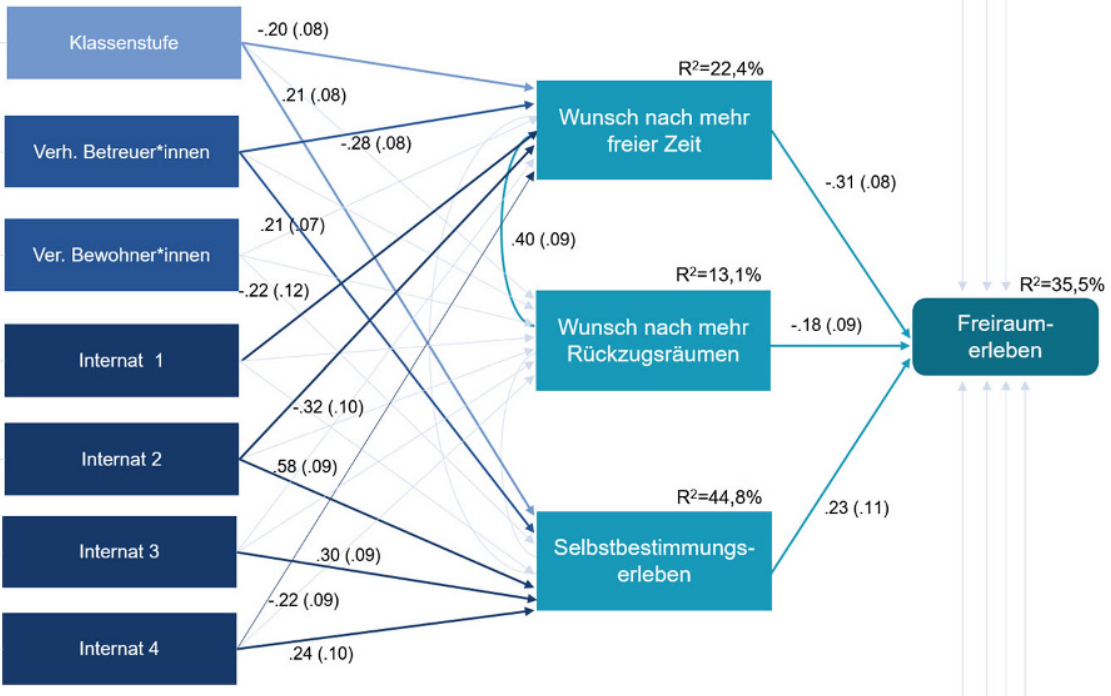

Abb. 3 Erweitertes Pfadmodell zur Erklärung von Freiraumerleben ( $n=113)$ (Standardisierte Koeffizienten (Standardfehler in Klammern); Hervorgehobene Pfade signifikant mit $p<0,05$ )

\section{Fazit}

Ein theoretisches Modell von Freiraumerleben gerade im Kontext von Internaten $\mathrm{zu}$ entwerfen und zu überprüfen, mag auf den ersten Blick irritieren, ist zumindest das öffentliche Bild von Internaten doch nicht unbedingt von großen Freiheiten für Jugendliche geprägt. Aber gerade die starke Prägung des Alltags der Jugendlichen durch die sehr umfassende Rahmung von Internaten nutzt der Beitrag, um sich dem Begriff Freiraum zu nähern. Dabei wird Freiraum als etwas Relationales verstanden, das sich vor allem über die jeweilige Wahrnehmung gegebener individueller Relationen zu Institutionen (zu Internaten, aber auch zu Familie, Schule, Vereinen etc.) bestimmt. Der in den empirischen Daten über die Kontextebene aufgeklärte Varianzanteil von sechs Prozent weist für das Freiraumempfinden dabei sowohl auf die gemeinsame Bedeutung des jeweiligen Internatskontexts als auch auf eine darin unterschiedliche Wahrnehmung hin.

Als wesentliche drei Dimensionen des Freiraumempfindens wurden theoretisch das Bedürfnis nach freien Zeiten, das Bedürfnis nach Rückzugsräumen sowie die erlebten Selbstbestimmungsmöglichkeiten entwickelt und empirisch geprüft. Mit einer Varianzaufklärung von über $33 \%$ bewährt sich diese modellhafte Vorstellung für den Kontext Internat. Weitere Einflussfaktoren auf das Freiraumempfinden wer- 
den über diese Dimensionen vermittelt: institutionelle/interaktionale (Verhältnis zu den Betreuer*innen und Mitbewohner*innen) sowie personenbezogene (Höhe der Klassenstufe, die mit mehr „Freiheitsrechten“ im Internat verbunden ist) Einflussfaktoren wirken nur indirekt auf das Freiraumempfinden ebenso wie die darüber hinausgehenden Effekte des jeweiligen Internatskontextes. Hier wäre mit Blick auf das pädagogische Setting eine nähere Analyse pädagogischer Konzeptionen und Praxis der Internate interessant, um den Internatseffekten konkreter nachzugehen.

Dies führt zu den Limitationen der Studie. Allein das begrenzte Sample von fünf Internaten stellt eine Herausforderung dar, da die geringe Fallzahl eine Modellierung komplexer Modelle nur begrenzt zulässt. Und gerade um den Einfluss der jeweiligen Internate über Mehrebenenanalysen näher zu untersuchen, wäre eine Einbeziehung von deutlich mehr Internaten wünschenswert. Auch der nicht für die hier skizzierte Forschungsfrage angelegte Fragebogen begrenzt die Analysemöglichkeiten. So wäre es wünschenswert, das Zeit- und Raumerleben komplexer operationalisieren oder eben die „Qualität“" pädagogischer Prozesse und Interaktionen stärker einbeziehen zu können.

Dennoch wird mit den vorliegenden Ergebnissen davon ausgegangen, dass das Modell sich nicht nur für das Heranwachsen in vergleichbar umfassenden Institutionen wie der stationären Erziehungshilfen bewährt, sondern auch auf ,klassisches“ Jugendleben übertragen werden kann.

Funding Open Access funding enabled and organized by Projekt DEAL.

Open Access Dieser Artikel wird unter der Creative Commons Namensnennung 4.0 International Lizenz veröffentlicht, welche die Nutzung, Vervielfältigung, Bearbeitung, Verbreitung und Wiedergabe in jeglichem Medium und Format erlaubt, sofern Sie den/die ursprünglichen Autor(en) und die Quelle ordnungsgemäß nennen, einen Link zur Creative Commons Lizenz beifügen und angeben, ob Änderungen vorgenommen wurden.

Die in diesem Artikel enthaltenen Bilder und sonstiges Drittmaterial unterliegen ebenfalls der genannten Creative Commons Lizenz, sofern sich aus der Abbildungslegende nichts anderes ergibt. Sofern das betreffende Material nicht unter der genannten Creative Commons Lizenz steht und die betreffende Handlung nicht nach gesetzlichen Vorschriften erlaubt ist, ist für die oben aufgeführten Weiterverwendungen des Materials die Einwilligung des jeweiligen Rechteinhabers einzuholen.

Weitere Details zur Lizenz entnehmen Sie bitte der Lizenzinformation auf http://creativecommons.org/ licenses/by/4.0/deed.de.

\section{Literatur}

Abels, I. (2006). Kinder zwischen Heimerziehung und Kinder- und Jugendpsychiatrie. Übergänge im Erleben der Betroffenen. Siegen: Universität Siegen.

Arbeitsgemeinschaft für Kinder- und Jugendhilfe (AGJ) (2016). Freiräume für Jugend schaffen! Diskussionspapier der AGJ. https:/www.agj.de/fileadmin/files/positionen/2016/Freir\%C3\%A4ume_f\%C3 $\%$ BCr_Jugend_schaffen.pdf. Zugegriffen: 30. Juni 2019.

Böhnisch, L. (2012). Lebensbewältigung. Ein sozialpolitisch inspiriertes Paradigma für die Soziale Arbeit. In W. Thole (Hrsg.), Grundriss Soziale Arbeit (S. 219-233). Wiesbaden: VS.

Bundesministerium für Verkehr, Bau und Stadtentwicklung (BMVBS) (2010). Werkstatt: Praxis Heft 70. Freiräume für Kinder und Jugendliche. Berlin. https://www.bbsr.bund.de/BBSR/DE/Veroeffentlichung en/ministerien/BMVBS/WP/2010/H70.html?nn=441864. Zugegriffen: 30. Juni 2019. 
Chin, W.W. (1998). The partial least squares approach for structural equation modeling. In G. A. Marcoulides (Hrsg.), Modern methods for business research (S. 295-336). London: Psychology Press.

Cohen, J. (1988). Statistical power analysis for the behavioral sciences. Hillsdale: Psychology Press.

Deci, E.L., \& Ryan, R. M. (1993). Die Selbstbestimmungstheorie der Motivation und ihre Bedeutung für die Pädagogik. Zeitschrift für Pädagogik, 39(2), 223-238.

Deinet, U. (2005). „Aneignung“ und „Raum“ - zentrale Begriffe des sozialräumlichen Konzeptes. In U. Deinet (Hrsg.), Sozialräumliche Jugendarbeit (S. 27-57). Wiesbaden: VS.

Derecik, A. (2012). Freiräume im Schulgebäude - Informelle Tätigkeiten von Heranwachsenden in den Pausen von Ganztagsschulen. Schulpädagogik heute, 3(6), 1-17.

Deußer, A., \& Nebelin, M. (2009). Einleitung: Die Vieldimensionalität der vierten Dimension. In M. Nebelin \& A. Deußer (Hrsg.), Was ist Zeit? (S. 7-18). Berlin: LIT.

Deutscher Bundesjugendring (DBJR) (2010). Selbstbestimmt und nicht verzweckt. Ehrenamtliches Engagement junger Menschen. Berlin. https://www.dbjr.de/fileadmin/user_upload/pdf-dateien/ Publikationen/Broschueren/DBJR_brosch_engagement_web.pdf. Zugegriffen: 1. Sept. 2018.

Deutscher Bundestag (2017). Der 15. Kinder- und Jugendbericht. Bundestagsdrucksache 18/11050. Berlin: Deutscher Bundestag.

Erikson, E.H. (1980). Jugend und Krise. Stuttgart: Klett-Cotta.

Ferchoff, W. (2006). Wandervogel, Jugend und Jugendkultur. In W. Faulstich (Hrsg.), Das erste Jahrzehnt (S. 117-134). München: Wilhelm Fink.

Gibson, A. (2017). Klassenziel Verantwortungselite. Eine Studie zu exklusiven, deutschen Internatsgymnasien und ihrer Schülerschaft. Wiesbaden: Springer.

Giesecke, H. (1981). Vom Wandervogel bis zur Hitlerjugend. Jugendarbeit zwischen Politik und Pädagogik. München: Juventa.

Glowatz-Frei, F. (2015). Schulfreiräume nachhaltig gestalten. In G. Opp \& A. Bauer (Hrsg.), Lebensraum Schule. Raumkonzepte planen - gestalten - entwickeln (S. 213-233). Stuttgart: Frauenhofer IRB.

Goffman, E. (1981). Asyle. Über die soziale Situation psychiatrischer Patienten und anderer Insassen. Frankfurt am Main: Suhrkamp.

Görtler, M. (2016). Zu Didaktik und Zeitforschung der politischen Bildung. In M. Görtler (Hrsg.), Politische Bildung und Zeit (S. 13-40). Wiesbaden: VS.

Haep, C. (2015). Das Internat als ,fürsorgliche' Institution. In C. Haep (Hrsg.), Grundfragen der Internatspädagogik: Theorie und Praxis (S. 123-134). Würzburg: Königshausen \& Neumann.

Helsper, W., Böhme, J., Kramer, R.-T., \& Lingkost, A. (2001). Schulkultur und Schulmythos. Opladen: VS.

Hurrelmann, K., \& Quenzel, G. (2016). Lebensphase Jugend. Eine Einführung in die sozialwissenschaftliche Jugendforschung. Weinheim: Beltz Juventa.

Kalthoff, H. (1997). Wohlerzogenheit. Eine Ethnografie deutscher Internatsschulen. Frankfurt am Main: Campus.

Katenbrink, N. (2014). Autonomie und Heteronomie: Peers und Schule. Das Beispiel eines reformpädagogischen Internats. Opladen: Budrich UniPress.

Keupp, H. (2003). Identitätskonstruktion. http://www.ipp-muenchen.de/texte/identitaetskonstruktion.pdf. Zugegriffen: 10. Juni 2019.

Kolbe, F.-U., Rabenstein, K., \& Reh, S. (2006). Expertise „Rhythmisierung“. Hinweise für die Planung von Fortbildungsmodulen für Moderatoren. https://www.ganztaegig-lernen.de/media/Material_1/ Expertise_Rhythmisierung.pdf. Zugegriffen: 2. Juli 2019.

Ladenthin, V. (2009). Enzyklopädisches Stichwort: Internat. In V. Ladenthin, H. Fitzek \& M. Ley (Hrsg.), Das Internat. Struktur und Zukunft. Ein Handbuch (S. 13-32). Würzburg: Ergon Verlag.

Langer, W. (2009). Mehrebenenanalyse. Eine Einführung für Forschung und Praxis. Wiesbaden: VS.

Löw, M. (2001). Raumsoziologie. Frankfurt am Main: Suhrkamp.

Maas, C., \& Hox, J. (2005). Sufficient sample sizes for multilevel modeling. Methodology: European Journal of Research Methods for the Behavioral and Social Sciences, 1(3), 86-92.

Opaschowski, H.W. (1994). Freizeitwissenschaft als neue Spektrumswissenschaft. In D. Benner \& D. Lenzen (Hrsg.), Bildung und Erziehung in Europa. 32. Beiheft der Zeitschrift für Pädagogik. (S. 441-444). Weinheim: Beltz.

Opaschowski, H. W. (2008). Einführung in die Freizeitwissenschaft. Wiesbaden: VS.

Peyerl, K. (2020). Jugend im Internat. Eine Untersuchung zum Erleben von Fremd-, Mit- und Selbstbestimmung im Spannungsverhältnis von Jugend- und Organisationsleben. Marburg: Dissertation. https:// archiv.ub.uni-marburg.de/diss/z2020/0096/pdf/dkp.pdf. Zugegriffen: 22. Apr. 2020.

Prahl, H.-W. (2015). Geschichte und Entwicklung der Freizeit. In R. Freericks \& D. Brinkmann (Hrsg.), Handbuch Freizeitsoziologie (S. 3-27). Wiesbaden: Springer. 
Reinders, H. (2003). Jugendtypen: Ansätze zu einer differentiellen Theorie der Adoleszenz. Opladen: Leske \& Budrich.

Rössler, B. (2001). Der Wert des Privaten. Frankfurt am Main: Suhrkamp.

Rössler, B. (2017). Autonomie. Berlin: Suhrkamp.

Soremski, R. (2013). Keine Zeit für Freizeit? Ganztagsschule im Alltag Jugendlicher. https://www.bmbf. de/files/keine_zeit_fuer_freie_zeit_bf_2.pdf. Zugegriffen: 25. Apr. 2019.

Tenorth, H.-E., \& Tippelt, R. (2012). Internatsformen. In H.-E. Tenorth \& R. Tippelt (Hrsg.), Beltz Lexikon Pädagogik (S. 349). Weinheim: Beltz.

Trescher, H. (2015). Die Würde des Privaten. Zur Diskussion institutionalisierter Lebensbedingungen von Menschen mit kognitiver Beeinträchtigung. Behindertenpädagogik, 54(2), 136-153.

Waldschmidt, A. (2012). Selbstbestimmung als Konstruktion. Alltagstheorien behinderter Frauen und Männer. Wiesbaden: VS.

Zinnecker, J. (2000). Kindheit und Jugend als pädagogische Moratorien. In D. Benner \& H.-E. Tenorth (Hrsg.), Bildungsprozesse und Erziehungsverhältnisse im 20. Jahrhundert. 42. Beiheft der Zeitschrift für Pädagogik (S. 36-68). Weinheim: Beltz.

Züchner, I., \& Peyerl, K. (2018). Aufwachsen am Lebensort Internat. In C. Haep, P. Koci, V. Ladenthin \& M.-T. Pütz-Böckem (Hrsg.), Gegenwart und Zukunft des Internats. Bestandsaufnahme und Forschungsaufgaben (S. 63-84). Bonn: Verlag für Kultur und Wissenschaft. 